\title{
含苯并噻二唑的二维有机染料的合成与光伏性能研究
}

\author{
曹镇财何 舟 邓利军 谭松庭* \\ (湘潭大学化学学院 教育部环境友好化学与应用重点实验室 湘潭 411105)
}

\begin{abstract}
摘要 通过引入烷基二噻吩苯并噻二唑窄带隙基元到 $\mathrm{D}-\pi-\mathrm{A}$ 有机染料体系中, 设计并合成了两种二维结构的有机染料 DD2 和 DH2, 系统地研究了以烷基二噻吩苯并噻二㟇基元作为侧基或桥联基元对染料敏化剂的光物理性能、电化学性 能和光伏性能的影响. 含有烷基二噻吩苯并噻二唑基元的这两种染料都具有较宽的吸收光谱. 在氙灯光源 (100 $\mathrm{mW} \cdot \mathrm{cm}^{-2}, \mathrm{AM} 1.5 \mathrm{G}$ ) 下, 基于有机染料 DH2 和 DD2 的染料敏化太阳能电池的能量转换效率分别为 $1.67 \%$ 和 $3.26 \%$. 采 用鹅去氧胆酸(CDCA)共吸附剂优化后, 染料 DH2 表现出较好的抗聚集能力, 能量转换效率几乎没有变化; 而 DD2 的 能量转换效率提高到了 $5.53 \%$.
\end{abstract}

关键词 有机染料; 苯并噻二唑; 合成; 染料敏化太阳能电池

\section{Synthesis and Photovoltaic Properties of Two Dimensional Organic Dyes Containing Benzothiadiazole Unit}

\author{
Cao, Zhencai He, Zhou Deng, Lijun Tan, Songting* \\ (Key Laboratory of Environmentally Friendly Chemistry and Applications of Ministry of Education, \\ College of Chemistry, Xiangtan University, Xiangtan 411105)
}

\begin{abstract}
By introducing the narrow band gap unit (dithiophene-benzothiadiazole) to the organic dye system, two novel organic dyes (DD2, DH2) with two-dimensional structures were designed and synthesized. The influence of dithiophene-benzothiadiazole unit acting as side chain or bridge group on the photophysical, electrochemical, and photovoltaic properties of the two dye-sensitizers was studied systematically. Two as-synthsized dyes with dithiophene-benzothiadiazole unit exhibited broad absorption spectra. Under one sun illumination $\left(100 \mathrm{~mW} \cdot \mathrm{cm}^{-2}\right.$, AM $\left.1.5 \mathrm{G}\right)$, DH2- and DD2-based dye-sensitized solar cells showed the power conversion efficiencies (PCE) of $1.67 \%$ and $3.26 \%$, respectively. With optimization of co-adsorbent chenodeoxycholic acid (CDCA), DH2 exhibited a relatvely good anti-aggregation ability. Therefore, CDCA had a negligible effect on the PCE of DH2-based DSSCs. However, DD2 achieved an enhanced PCE of 5.53\% after adding CDCA as co-adsorbent.
\end{abstract}

Keywords organic dyes; benzothiadiazole, synthesis; dye-sensitized solar cells

1991 年, Grätzel 等 ${ }^{[1]}$ 首次提出了染料敏化太阳能电 池(DSSCs)的结构模型. 由于这类染料敏化太阳能电池 的成本低廉，制作工艺简单，可设计性强，应用潜力大， 因而引起了研究工作者的极大兴趣 ${ }^{[2 \sim 6]}$. 近年来, 该领 域的研究取得了重要进展, 当前的染料敏化电池效率已 高达 $12.3 \%{ }^{[7]}$, 这与多晶硅太阳能电池的效率已经很接 近.

目前, 以 $\mathrm{N} 79^{[8]}, \mathbf{C O 1}^{[9]}, \mathbf{N} 3^{[10]}$, 黑染料 ${ }^{[11]}$ 等为代表 的染料的能量转换效率(PCE)均已接近甚至超过了 $10 \%$,
但它们的分子结构中含有贵金属钉 $(\mathrm{Ru})$, 限制了其广泛 应用. 研究开发不含贵金属的有机染料成为该领域的一 个重要研究方向. 三苯胺及其衍生物本身具有 3D 螺旋 星型结构、优良的供电子及传输电荷的能力，已经作为 有机染料敏化剂的基元成功地应用于 DSSCs 中. 2011 年, 王鹏等 ${ }^{[12]}$ 设计并合成了含三苯胺结构的纯有机染 料 C219，最高效率达到了 $10.3 \%$. 国内一些研究组在有 机染料敏化剂的合成与光伏性能研究方面也取得了较 好的研究进展 ${ }^{[13 \sim 18]}$. 在有机染料的结构设计方面, 为了

\footnotetext{
*E-mail: tanst2008@163.com, Tel./Fax: 0086-731-58292207.

Received September 3, 2013; revised October 11, 2013; published online October 31, 2013.

Project supported by the National Natural Science Foundation of China (No. 51173154) and the Natural Science Foundation of Hunan Province (No. 13JJ2025).

国家自然科学基金(No. 51173154)和湖南省自然科学基金(No. 13JJ2025)资助项目.
} 
增加针定基团, 扩大共轭体系, 大量的研究工作开始由 传统的 D- $\pi$-A 结构向复杂拓扑结构发展, 因为复杂拓扑 结构可以使共轭体系增大, 同时进一步增加了分子的可 设计性, 还能有效地降低分子的聚集. 近期出现了一些 $\Pi$ 型结构 ${ }^{[19 ~ 21]}$ 和 $Y$ 型结构 ${ }^{[22,23]}$ 的有机染料, Heredia 等 ${ }^{[24]}$ 设计合成了十字状交叉的三维结构的染料. 2012 年, $\mathrm{Li}$ 等 ${ }^{[25]}$ 设计合成了 $\mathrm{H}$ 型结构的染料, 复杂的空间结 构有效的降低了染料的聚集，同时获得了较高的效率.

我们 ${ }^{[26]}$ 曾报道了具有三苯胺一塞吩-氧基乙酸 D- $\pi$-A 体系的有机染料 H1 具有较好的光伏性能, 但该 有机染料吸收光谱范围较窄; 为拓宽有机染料的吸收光 谱, 本文将烷基二噻吩苯并噻二唑窄带隙基元作为侧基 引入 $\mathrm{D}-\pi-\mathrm{A}$ 体系中, 设计并合成了二维 $\mathrm{T}$ 型结构有机染 料 DD2; 同时为了进一步扩大共轭体系, 在此基础上以 烷基二噻吩苯并噻二唑为桥联基元，设计合成了二维 $\mathrm{H}$ 型结构的有机染料 DH2(结构式见 Scheme 1), 研究和比 较了烷基二噻吩苯并噻二唑基元作为侧基或桥联基元 对染料敏化剂的光物理性能、电化学性能和光伏性能的 影响.

\section{1 结果与讨论}

\section{1 有机染料的光物理性质}

图 1(a) 是 3 个有机染料在氯仿稀溶液 $\left(10^{-5} \mathrm{~mol} / \mathrm{L}\right)$ 中 的紫外-可见吸收光谱, DD2 和 DH2 都表现出典型的双 带吸收行为, 分别出现在 $300 \sim 480$ 和 $500 \sim 650 \mathrm{~nm}$ 处; 其中在 $300 \sim 480 \mathrm{~nm}$ 范围内的吸收带归属于共轭分子的 $\pi-\pi *$ 电子转移跃迁, 在 $500 \sim 650 \mathrm{~nm}$ 范围内的吸收带可
以归属于电子给体和氧基乙酸电子受体之间的电荷转 移 $(\mathrm{ICT})^{[27]}$. 从图 1(a)中可以看出, DH2 的 ICT 的最大吸 收峰比 DD2 有着较大程度的红移, 可归因于共轭体系 增大, 能隙减小. DD2 在 385 和 $520 \mathrm{~nm}$ 两个吸收峰处的 摩尔吸收系数分别为 $6.5 \times 10^{4}$ 和 $5.7 \times 10^{4} \mathrm{~L}^{\circ} \mathrm{mol}^{-1} \cdot \mathrm{cm}^{-1}$, DH2 在 445 和 $562 \mathrm{~nm}$ 两个吸收峰处的摩尔吸收系数分 别为 $4.0 \times 10^{4}$ 和 $6.1 \times 10^{4} \mathrm{~L} \cdot \mathrm{mol}^{-1} \cdot \mathrm{cm}^{-1}$. 两个有机染料 的摩尔吸收系数均大于 $\mathrm{N} 719\left(\varepsilon=1.4 \times 10^{4} \mathrm{~L} \cdot \mathrm{mol}^{-1}\right.$. $\left.\mathrm{cm}^{-1}\right)^{[28]}$, 这表明它们均有较强的光吸收能力. 有机染 料 DD2 和 DH2 与我们之前报道的染料 $\mathrm{H1}$ 相比 ${ }^{[26]}$, 吸 收峰都有明显的红移和加宽, 摩尔吸收系数也有 $2 \sim 4$ 倍的增加, 表明烷基二噻吩苯并噻二唑窄带隙基元有效 地改善了有机染料对光的吸收能力.

表 1 有机染料 DD2, DH2 和 $\mathrm{H1}$ 的光物理性能

Table 1 Photophysical properties of the organic dyes DD2, DH2 and H1

\begin{tabular}{cccc}
\hline Dyes & \multicolumn{2}{c}{$\lambda_{\text {abs }}{ }^{a} / \mathrm{nm}\left[\varepsilon /\left({\left.\left.\mathrm{L} \cdot \mathrm{mol}^{-1} \bullet \mathrm{cm}^{-1}\right)\right]} \lambda_{\mathrm{em}}{ }^{b} / \mathrm{nm}\right.\right.$} \\
\hline DD2 & $385\left(6.5 \times 10^{4}\right)$ & $520\left(5.7 \times 10^{4}\right)$ & 685 \\
DH2 & $445\left(4.0 \times 10^{4}\right)$ & $562\left(6.1 \times 10^{4}\right)$ & 695 \\
H1 & $484\left(2.6 \times 10^{4}\right)$ & $363\left(1.3 \times 10^{4}\right)$ & 645 \\
\hline
\end{tabular}

${ }^{a}$ The maximum absorption in $\mathrm{CHCl}_{3}$ solution $\left(10^{-5} \mathrm{~mol} / \mathrm{L}\right) \cdot \varepsilon$ is the molar absorption coefficient at maximum absorption. ${ }^{b}$ The maximum emission peak in $\mathrm{CHCl}_{3}$ solution $\left(10^{-5} \mathrm{~mol} / \mathrm{L}\right)$.

我们还测试了有机染料在薄膜上的 UV-Vis 吸收光 谱, 如图 1(b)所示. 从图中可以看出, 有机染料 DH2 和 DD2 在固体膜中的吸收范围相对于溶液中的都有一定 程度的红移, 这是由于染料分子存在 $J$-型聚集的缘 故 ${ }^{[29]}$.
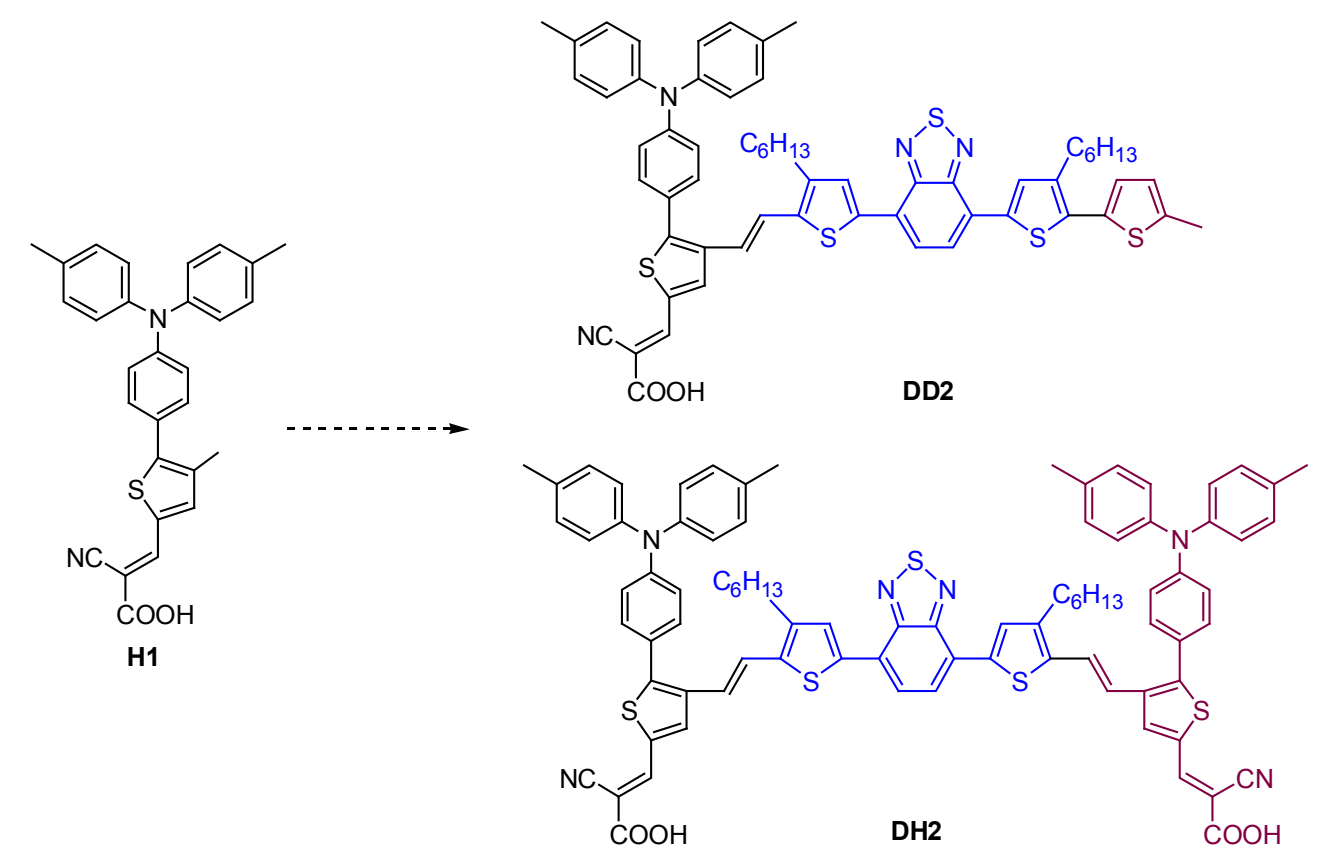

Scheme 1 

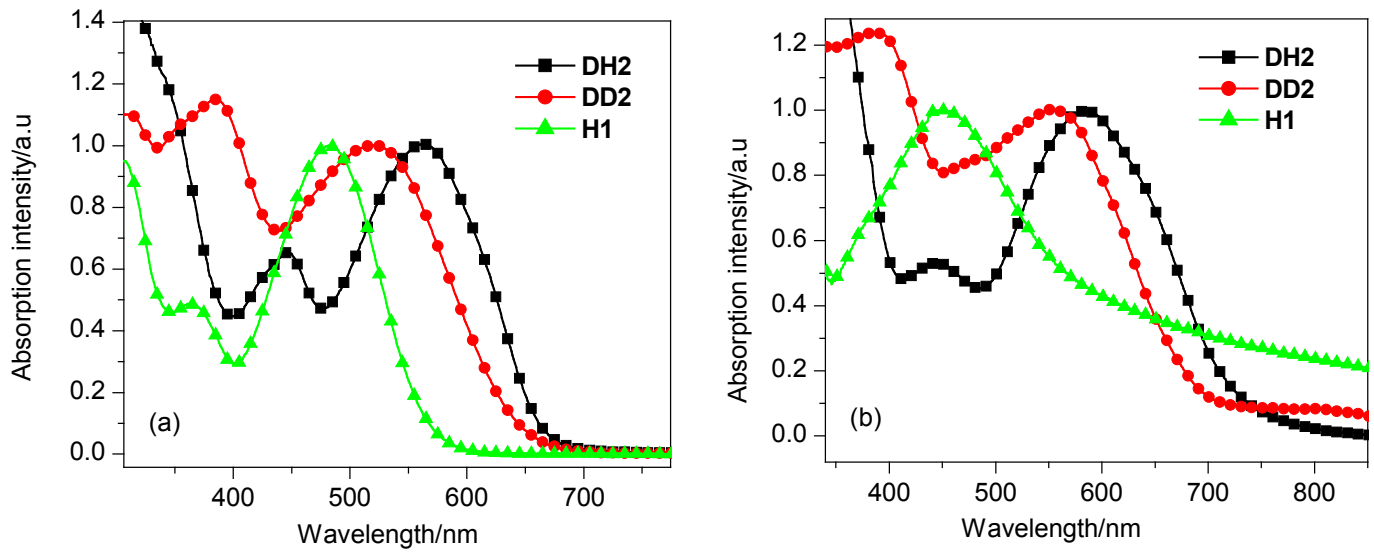

图 1 有机染料 DD2, DH2 和 H1 的 UV-Vis 光谱

Figure 1 UV-Vis absorption spectra of organic dyes DD2, DH2 and H1 (a) In $\mathrm{CHCl}_{3}$ solution and (b) on $\mathrm{TiO}_{2}$ film

\section{2 有机染料的电化学性质}

为了研究有机染料分子的电荷注入和还原再生能 力, 我们以二氯甲烷为溶剂, 四丁基六氟磷酸铵(TBAP) 为电解质, 利用循环伏安法 $(\mathrm{CV})$ 测定了 DH2 和 DD2 的 电化学性质, 所得循环伏安曲线如图 2 所示. 以二茂铁 $\left(\mathrm{Fc} / \mathrm{Fc}^{+}\right)$为内标, 其氧化还原电位为 $0.63 \mathrm{~V}$ (vs. NHE), 图 2 中有机染料第一氧化电位与二茂铁 $\left(\mathrm{Fc} / \mathrm{Fc}^{+}\right)$的电位 差, 再加上二茂铁 $\left(\mathrm{Fc} / \mathrm{Fc}^{+}\right)$的氧化电位 $0.63 \mathrm{~V}$ (vs. NHE), 即为染料激发态相对于标准氢电极(NHE) 的氧化电位 $E_{\mathrm{ox}}$. 染料激发态氧化电势 $E_{\mathrm{ox}}{ }^{*}$ 与 $E_{\mathrm{ox}}$ 满足以下关系:

$$
E_{\mathrm{ox}}{ }^{*}=E_{\mathrm{ox}}-E_{0-0}
$$

$E_{0-0}$ 是指染料的基态跃迁能, 它是根据染料在溶液中的 归一化紫外-可见吸收光谱和荧光光谱交叠处的波长 $\lambda_{\mathrm{int}}$ 计算得到的. 然后, 可以根据上述公式计算得到染料的 $E_{\mathrm{ox}}{ }^{*}$, 两种有机染料的电化学数据见表 2 . 从表 2 中可以 看到, 一方面, 染料 DD2 和 $\mathbf{D H 2}$ 的 $E_{\mathrm{ox}}{ }^{*}$ 分别为 -1.05 和 $-0.89 \mathrm{~V}$, 均比 $\mathrm{TiO}_{2}$ 的导带能级 $(-0.50 \mathrm{~V}$ vs. NHE)小 得多, 这表明所合成的 2 种有机染料的激发态电子能迅 速注入到 $\mathrm{TiO}_{2}$ 导带, 并且从电子注入能力 $\left(\Delta G_{\mathrm{inj}}\right)$ 来看, DD2 更有利于电子的传输. 另一方面, 染料 DD2 和 DH2 的 $E_{\text {ox }}$ 分别为 0.97 和 $0.95 \mathrm{~V}$, 均比 $\mathrm{I}^{-} / \mathrm{I}_{3}{ }^{-}$的氧化电位 $\left(0.5 \mathrm{~V}\right.$ vs. NHE) 要大很多, 即 $\Delta G_{\mathrm{reg}}$ 较大, 这说明氧化态 染料分子可被电解质溶液中的 $\mathrm{I}^{-}$有效地还原再生. 综上 所述，两种有机染料均能有效的作为染料敏化剂应用于
DSSCs 中, 且 DD2 的能级更合适，有可能获得更高的光 电转换效率.

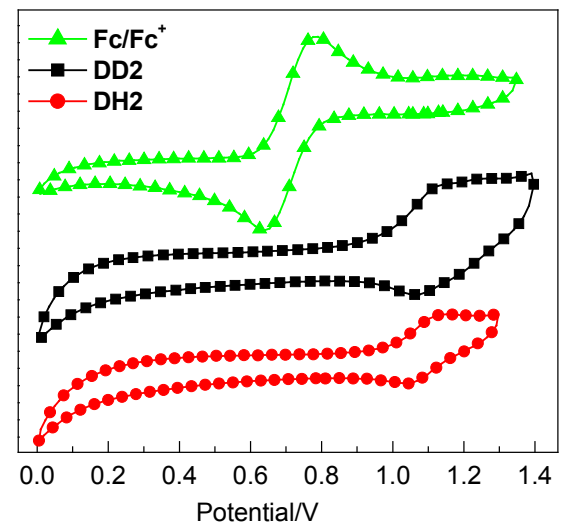

图 2 有机染料 DD2, DH2 和二茂铁的循环伏安曲线

Figure 2 Cyclic voltammogram of organic dyes DD2, DH2 and ferrocene

Scan conditions: Pt electrodes for work and counter electrode, calomel electrode for reference electrode, and a scan rate of $100 \mathrm{mV} / \mathrm{s}$, ferrocene as inner reference

\section{3 有机染料的光伏性能}

我们测试了 2 个有机染料敏化剂(DH2 和 DD2)应用 于 DSSCs 的光伏性能. 图 3a 是基于二个染料敏化剂所 制得 DSSC 器件的入射单色光子-电子转化效率 (IPCE) 与波长关系曲线. 从图中可以看出，几乎在整个可见光 区, DD2 的 IPCE 值都比 DH2 高, 意味着染料 DD2 的短

表 2 有机染料 DD2 和 DH2 的电化学性能

Table 2 Electrochemical properties of organic dyes DD2 and DH2

\begin{tabular}{cccccccc}
\hline Dye & $\lambda_{\text {int }}{ }^{a} / \mathrm{nm}$ & $E_{0-0}{ }^{b} / \mathrm{eV}$ & $E_{\mathrm{o}} / \mathrm{V}$ vs. NHE & $E_{\text {ox }}{ }^{*} \mathrm{c} / \mathrm{V}$ vs. NHE & $\Delta G_{\text {inj }}{ }^{d} / \mathrm{eV}$ & $\Delta G_{\text {reg }}{ }^{e} / \mathrm{eV}$ \\
\hline DD2 & 614 & 2.02 & 0.97 & -1.05 & -0.55 & -0.47 \\
DH2 & 673 & 1.84 & 0.95 & -0.89 & -0.39 & -0.45 \\
\hline
\end{tabular}

${ }^{a}$ Determined from the intercept of the normalized absorption and emission spectra. ${ }^{b}$ Bandgap, $E_{0-0}=1240 / \lambda_{\text {int. }}{ }^{c}$ Excited-state oxidation potentials approximated from $E_{\mathrm{ox}}$ and $E_{0-0}$ (vs. NHE). $E_{\mathrm{ox}}{ }^{*}=E_{\mathrm{ox}}-E_{0-0 .}{ }^{d}$ Driving forces for electron injection from the dye excited singlet state $\left(E_{\mathrm{ox}}{ }^{*}\right)$ to the conduction band of TiO ${ }_{2}(-0.5 \mathrm{~V}$ vs. NHE). $\Delta G_{\mathrm{inj}}=E_{\mathrm{ox}}{ }^{*}-(-0.5 \mathrm{~V}) .{ }^{e}$ Driving forces for regeneration of the dye radical cation $\left(E_{\mathrm{ox}}\right)$ by $\mathrm{I}^{-} / \mathrm{I}_{3}{ }^{-}$redox couple $(0.5 \mathrm{~V} \mathrm{vs} . \mathrm{NHE}) . \Delta G_{\mathrm{reg}}=0.5 \mathrm{~V}-E_{\mathrm{ox}}$. 
表 3 基于有机染料 DD2 和 DH2 的 DSSCs 的光伏性能

Table 3 Photovoltaic performance of DSSCs based on dyes DD2 and DH2

\begin{tabular}{lccccc}
\hline \multicolumn{1}{c}{ Dye } & $J_{\text {sc }} /\left(\mathrm{mA} \cdot \mathrm{cm}^{-2}\right)$ & $V_{\text {oc }} / \mathrm{V}$ & FF & PCE $/ \%$ & Adsorbed amount of dye $/\left(10^{-8} \mathrm{~mol} \cdot \mathrm{cm}^{-2}\right)$ \\
\hline DD2 & 7.39 & 0.65 & 0.68 & 3.26 & 2.07 \\
DD2 + CDCA & 12.20 & 0.64 & 0.71 & 5.56 & - \\
DH2 & 4.23 & 0.55 & 0.72 & 1.67 & 0.95 \\
DH2 + CDCA & 4.02 & 0.55 & 0.69 & 1.53 & - \\
\hline
\end{tabular}
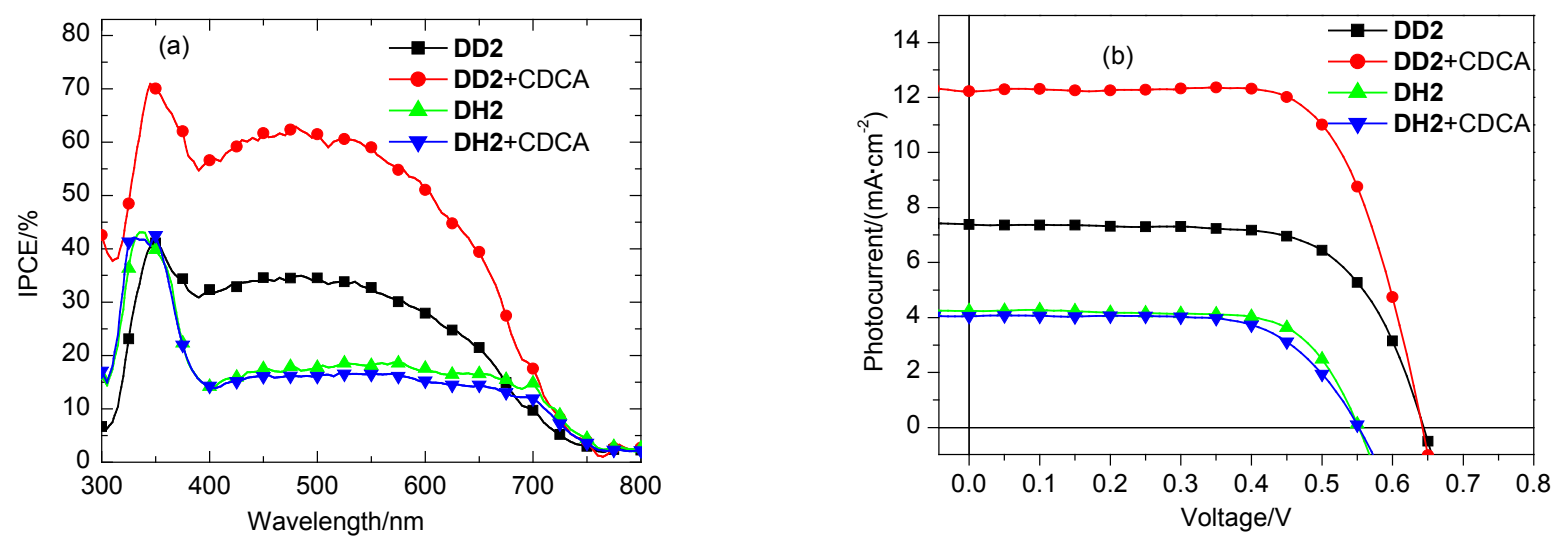

图 3 基于有机染料 DD2 和 DH2 的 DSSCs 的(a) IPCE 曲线和(b) $J-V$ 曲线

Figure 3 IPCE curve (a) and $J-V$ curve (b) of DSSCs sensitized by organic dyes DD2 and DH2

路电流 $J_{\mathrm{sc}}$ 将会比较高. 同时, 与它们的 UV-Vis 光谱图 相对应, DH2 的 IPCE 光谱比 DD2 稍有的拓宽. 图 $3 b$ 是 在 AM1.5 模拟太阳光 $\left(100 \mathrm{~mW} \cdot \mathrm{cm}^{-2}\right)$ 照射下基于染料 DH2 和 DD2 的 DSSCs 的 $J-V$ 曲线, 相应的光伏性能数 据列于表 3 中. 电池的 $J_{\mathrm{sc}}$ 值与其 IPCE 曲线的积分面积 有直接的关系 ${ }^{[30]}$, IPCE 曲线积分面积越大, $J_{\mathrm{sc}}$ 值越大. 从 DH2 和 DD2 的 IPCE 测试结果可知, DD2 在紫外和可 见光范围的 IPCE 曲线积分面积相对较大, 因此应当具 有更高的 $J_{\mathrm{sc}}$ 值. 从表 3 中我们可以看到 DD2 的 $J_{\mathrm{sc}}$ 值要 明显高于 DH2, 这一结果与上述染料的 IPCE 测试结果 相符.

在相同的测试条件下, 基于有机染料 DD2 和 DH2 的 DSSCs 的能量转换效率值(PCE) 分别为 $1.67 \%$ 和 $3.26 \%$, DD2 的能量转换效率要明显高于 DH2. 这一结 果是由于 DD2 具有更高的开路电压与短路电流所致. 究其原因, 可能来自两方面: 首先, 开路电压与染料的 能级有关; DD2 相比 DH2, 其激发态氧化电势 $E_{\mathrm{ox}}{ }^{*}$ 与 $\mathrm{TiO}_{2}$ 导带能级差更大, 更有利于电子向 $\mathrm{TiO}_{2}$ 的注入, 因 而具有更高的开路电压 ${ }^{[31,32]}$. 其次, 电池的短路电流在 一定程度上受染料吸附量的影响 ${ }^{[33]}$, 两染料的摩尔吸 光系数相当, 但从表 3 可以看出 DD2 的吸附量明显高于 DH2. 很大程度上解释了 DD2 短路电流较高的原因. DH2 的吸附量较低是由于多引入一个三苯胺结构后, 分子的空间位阻增大, 影响了分子在 $\mathrm{TiO}_{2}$ 纳米晶膜上 的吸附.

加入共吸附剂 CDCA 优化后, DD2 的能量转换效率
进一步增加至 5.56\%，而 DH2 反而稍有下降. 对于 DD2, 共敏化剂的加入使短路电流大幅提高，说明共敏化剂抑 制了染料的聚集，从而能够更有效的实现激子的分离和 电荷的有效传输. 而对于 DH2, 分子空间结构复杂, 分 子中有两个非平面的三苯胺结构，空间位阻大，本身有 效地抑制了染料的聚集，加入 CDCA 后没有明显的变化 也说明了这一点.

\section{2 总结}

将烷基二噻吩-苯并噻二唑作为侧基或桥联基元引 入到三苯胺类 $\mathrm{D}-\pi-\mathrm{A}$ 有机染料体系中, 设计并合成了两 种二维结构有机染料敏化剂 DD2 和 DH2, 并研究比较 了其光物理、电化学和光伏性能. 研究结果表明, 引入 烷基二噻吩一苯并噻二唑基团后，两种染料都具有较宽 的吸收光谱和高的摩尔吸收系数, 有利于电池效率的提 高. 其中 $\mathrm{T}$ 型染料 DD2 的能量转换效率通过加入共吸附 剂 CDCA 优化后达到 5.56\%, 而具有 $\mathrm{H}$ 型二维结构的染 料 DH2 能量转换效率稍低. 这是因为, 与 DD2 相比, DH2 虽然具有较大的共轭结构, 导致光谱红移, 有利于 光的吸收, 但随着分子体积变大, 分子的空间位阻增大, 降低了染料在 $\mathrm{TiO}_{2}$ 膜上的吸附量，从而使效率有所降 低.

\section{3 实验部分}

\section{1 主要仪器及试剂}

核磁共振光谱在 Bruker ARX400 (400 MHz)核磁共 
振仪上进行，质谱在 Brucker Autoflex III 型飞行时间质 谱仪仪上进行, 红外光谱和紫外-可见光谱采用 PE 公司 的 Spectra One 型红外光谱仪和 PE Lamda 25 型紫外可 见光谱仪测试, 氧化还原电位采用 EG\&G Princeton Applied Research Model 273 型电化学工作站测定, 电池 的 J-V 曲线和入射单色光子-电子转化效率(IPCE)使用 Keithley 2602 和 Zolix Omni- $\lambda 150$ 单色仪进行测量.

试剂均为市售分析纯或化学纯, $N, N$-二甲基甲酰胺 用氢化钙回流处理, 四氢呋喃用钠丝回流, 然后常压蒸 馏, 其它试剂未经进一步提纯. 5-[7-(5-澳-4-已基-2-噻吩 基)-苯并 $[1,2,5]$ 噻二唑-4-基]-3-已基-2-噻吩基甲醛 (1) ${ }^{[34]} ， 2$-(三丁基锡基)-5-甲基噻吩(2) ${ }^{[35]} ， 5$-溴-3-(二乙 基-磷酸酯-甲基)-噻吩 (4) ${ }^{[36]}$, 频哪醇-[4-( $N, N$-二对甲苯 胺基)-1-苯基]硼酸酯(化合物 6) ${ }^{[28]}, 4,7$-二(4-己基噻吩-2基)苯并噻二唑(9) ${ }^{[36]}$ 根据文献合成.

\section{2 有机染料敏化剂的合成}

有机染料敏化剂 DD2 和 DH2 的合成路线如 Scheme 2 所示.

3.2.15-[7-(5-(5-甲基-2-噻吩基)-4-已基-2-噻吩基)苯并 $[1,2,5]$ 噻二唑-4-基]-3-已基-2-噻吩基甲醛(3)的合 成

向 $100 \mathrm{~mL}$ 三口瓶中依次加入化合物 $\mathbf{1}(0.70 \mathrm{~g}, 1.20$ $\mathrm{mmol})$, 化合物 $2(0.71 \mathrm{~g}, 1.83 \mathrm{mmol})$ 和甲苯 $(50 \mathrm{~mL})$, 抽 真空, 向反应体系通氩气 $30 \mathrm{~min}$, 接着向反应瓶中加入 $0.1 \mathrm{~g}$ 的 $\mathrm{Pd}\left(\mathrm{PPh}_{3}\right)_{4}$, 继续通氩气 $3.5 \mathrm{H}$ 然后将整个反应 体系的温度升至 $98{ }^{\circ} \mathrm{C}$, 反应 $3 \mathrm{~d}$. 待体系冷却至室温, 向体系中加入 $50 \mathrm{~mL}$ 蒸馏水, 再用二氯甲烷萃取三次, 水洗三次, 用无水硫酸镁干燥过夜, 蒸干溶剂, 粗产物 用 $[V$ (石油醚 $): V($ 二氯甲烷 $)=1: 1]$ 为洗脱剂过硅胶柱, 蒸发溶剂并真空干燥后得红褐色固体 $0.65 \mathrm{~g}$, 产率 $85 \%$. ${ }^{1} \mathrm{H}$ NMR $\left(\mathrm{CDCl}_{3}, 400 \mathrm{MHz}\right) \delta: 10.10$ (s, 1H, CHO), 8.06 (d, $J=4 \mathrm{~Hz}, 1 \mathrm{H}$, phenyl-H), $8.03(\mathrm{~d}, J=4 \mathrm{~Hz}, 1 \mathrm{H}$, phenyl-H), $795 \sim 7.97$ (s, $1 \mathrm{H}$, thienyl-H), $7.84 \sim 7.86$ (s, $1 \mathrm{H}$, thienyl-H), $7.04 \sim 7.05(\mathrm{~s}, 1 \mathrm{H}$, thienyl-H), $6.76(\mathrm{~s}, 1 \mathrm{H}$, thienyl-H), 3.02 (t, $\left.J=14 \mathrm{~Hz}, 2 \mathrm{H}, \mathrm{CH}_{2}\right), 2.83(\mathrm{t}, J=12 \mathrm{~Hz}$, $\left.2 \mathrm{H}, \mathrm{CH}_{2}\right), 2.54\left(\mathrm{~s}, 3 \mathrm{H}, \mathrm{CH}_{3}\right), 1.71 \sim 1.79\left(\mathrm{~m}, 4 \mathrm{H}, \mathrm{CH}_{2}\right)$, $1.34 \sim 1.43\left(\mathrm{~m}, 12 \mathrm{H}, \mathrm{CH}_{2}\right), 0.91\left(\mathrm{t}, J=12 \mathrm{~Hz}, 6 \mathrm{H}, \mathrm{CH}_{3}\right)$.

3.2 22-㴡-3-[2-[5-[7-(5-(5-甲基-2-噻吩基)-4-已基-2噻吩基)-苯并 $[1,2,5]$ 塞二唑-4-基]-3-已基-2-噻吩基]亚 乙烯基]塞吩(5)的合成

在 $50 \mathrm{~mL}$ 的单口瓶中, 依次加入化合物 $3(0.63 \mathrm{~g}$, $1.06 \mathrm{mmol})$, 化合物 $4(0.33 \mathrm{~g}, 1.06 \mathrm{mmol})$ 和 $15 \mathrm{~mL}$ 新蒸 的 THF. 然后, 在氩气保护和冰洗浴条件下向单口瓶中 逐滴滴加 $15 \mathrm{~mL}$ 溶有叔丁醇钾 $(0.21 \mathrm{~g}, 2.12 \mathrm{mmol})$ 的 $\mathrm{THF}$ 溶液. 室温反应 $5 \mathrm{H}$ 缓慢升温 $50{ }^{\circ} \mathrm{C}$ 回流 $1 \mathrm{~d}$, 待反
应体系冷却至室温，接着用二氯甲烷萃取 3 次，有机相 用蒸馏水洗涤，并用无水硫酸镁干燥，过滤，旋干溶剂 后得到粗产物, 粗产物用 $[V$ (石油醚) $: V$ (二氯甲烷 $)=$ $1: 1]$ 作洗脱剂进行硅胶柱层析纯化后得深褐色固体 $0.52 \mathrm{~g}$, 产率 65\%. ${ }^{1} \mathrm{H} \mathrm{NMR}\left(\mathrm{CDCl}_{3}, 400 \mathrm{MHz}\right) \delta: 7.98(\mathrm{~d}$, $J=4 \mathrm{~Hz}, 2 \mathrm{H}$, phenyl-H), 7.83 (d, $J=8 \mathrm{~Hz}, 2 \mathrm{H}$, thienyl-H), 7.22 (d, $J=15 \mathrm{~Hz}, 2 \mathrm{H}$, thienyl-H), 7.16 (s, $1 \mathrm{H}$, thienyl-H), $6.2(\mathrm{~d}, J=14 \mathrm{~Hz}, 2 \mathrm{H}, \mathrm{CH}), 6.75$ (s, 1H, thienyl-H), 2.82 (t, $\left.J=15 \mathrm{~Hz}, 2 \mathrm{H}, \mathrm{CH}_{2}\right), 2.74\left(\mathrm{t}, J=15 \mathrm{~Hz}, 2 \mathrm{H}, \mathrm{CH}_{2}\right), 2.53(\mathrm{~s}$, $\left.3 \mathrm{H}, \mathrm{CH}_{3}\right), 1.70 \sim 1.73\left(\mathrm{~m}, 4 \mathrm{H}, \mathrm{CH}_{2}\right), 1.25 \sim 1.42(\mathrm{~m}, 12 \mathrm{H}$, $\left.\mathrm{CH}_{2}\right), 0.91\left(\mathrm{t}, J=12 \mathrm{~Hz}, 6 \mathrm{H}, \mathrm{CH}_{3}\right)$.

3.2.3 (E)-4-(3-(2-(3-已基-5-(7-(5'-甲基-3-已基-[2,2'二塞吩]-5-基)苯并 [1,2,5]塞二唑-4-基)噻吩-2-基)亚乙 烯基)噻吩-2-基)- $N, N$-二对甲苯基胺(7)的合成

向 $100 \mathrm{~mL}$ 三口瓶中依次加入化合物 $5(0.48 \mathrm{~g}, 0.64$ $\mathrm{mmol})$, 化合物 $\mathbf{6}(0.31 \mathrm{~g}, 0.77 \mathrm{mmol}), 30 \mathrm{~mL}$ 新蒸的甲苯, $5 \mathrm{~mL}(2 \mathrm{~mol} / \mathrm{L}) \mathrm{K}_{2} \mathrm{CO}_{3}$ 水溶液, 在氩气保护下, 加入 100 $\mathrm{mg}$ 的 $\mathrm{Pb}\left(\mathrm{PPh}_{3}\right)_{4}(0.08 \mathrm{mmol})$, 升温至 $100{ }^{\circ} \mathrm{C}$ 反应 $72 \mathrm{~h}$ 后停止反应, 再将混合物倒入 $50 \mathrm{~mL}$ 蒸馏水中, 接着用 二氯甲烷萃取, 水洗二氯甲烷层, 无水硫酸镁干燥有机 层, 之后旋转蒸发溶剂, 粗产物用体积比为 $1: 3$ 的石油 醚/二氯甲烷作为淋洗剂进行硅胶柱层析, 得深褐色固 体 $0.49 \mathrm{~g}$, 产率 $81 \% .{ }^{1} \mathrm{H}$ NMR $\left(\mathrm{CDCl}_{3}, 400 \mathrm{MHz}\right) \delta: 7.98$ (d, $J=4 \mathrm{~Hz}, 2 \mathrm{H}$, phenyl-H), $7.80(\mathrm{~d}, J=8 \mathrm{~Hz}, 2 \mathrm{H}$, thienyl-H), 7.38 (d, $J=5 \mathrm{~Hz}, 2 \mathrm{H}$, thienyl-H), $7.14(\mathrm{~s}, 1 \mathrm{H}$, thienyl-H), 7.34 (d, $J=8 \mathrm{~Hz}, 2 \mathrm{H}, \mathrm{CH}), 7.09$ (d, $J=10 \mathrm{~Hz}$, $12 \mathrm{H}$, phenyl-H), 6.75 (d, $J=2 \mathrm{~Hz}, 1 \mathrm{H}$, thienyl-H), 2.82 (t, $\left.J=16 \mathrm{~Hz}, 2 \mathrm{H}, \mathrm{CH}_{2}\right), 2.73\left(\mathrm{t}, J=15 \mathrm{~Hz}, 2 \mathrm{H}, \mathrm{CH}_{2}\right), 2.53$ (s, $\left.3 \mathrm{H}, \mathrm{CH}_{3}\right), 2.33\left(\mathrm{~s}, 6 \mathrm{H}, \mathrm{CH}_{3}\right), 1.69 \sim 1.73\left(\mathrm{~m}, 4 \mathrm{H}, \mathrm{CH}_{2}\right)$, $1.34 \sim 1.42\left(\mathrm{~m}, 12 \mathrm{H}, \mathrm{CH}_{2}\right), 0.90\left(\mathrm{t}, J=12 \mathrm{~Hz}, 6 \mathrm{H}, \mathrm{CH}_{3}\right)$;

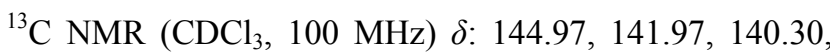
$139.96,134.42,133.05,130.77,130.57,130.19,125.99$, $125.89,125.70,125.35,125.15,123.83,122.55,121.65$, $120.51,31.72,31.70,30.93,30.63,29.69,29.50,29.31$, 29.17, 28.67, 22.65, 20.86, 15.32, 14.09; MALDI-TOF MS calcd for $\mathrm{C}_{57} \mathrm{H}_{57} \mathrm{~N}_{3} \mathrm{~S}_{5}(\mathrm{M}+\mathrm{H})^{+}$: 944.323 , found 944.319 .

3.2.4 (E)-5-[4-(二对苯甲基胺基)苯基]-4-(2-(3-已基5-(7-(3-已基-5'-甲基-[2,2'-二噻吩]-5-基)苯并 $[c][1,2,5]$ 噻二唑-4-基)噻吩-2-基)亚乙烯基)噻吩-2-甲醛(8)的合 成

在氩气保护和室温条件下, 于 $100 \mathrm{~mL}$ 两口瓶中注 入溶解在 $27 \mathrm{~mL}$ 新蒸 1,2-二氯乙烷中的化合物 $7(0.40 \mathrm{~g}$, $0.42 \mathrm{mmol})$, 然后在冰浴下分别加入 DMF $(0.33 \mathrm{~mL}, 4$ $\mathrm{mmol})$ 和 $\mathrm{POCl}_{3}(0.22 \mathrm{~mL}, 2.4 \mathrm{mmol})$. 继续搅拌 $5 \mathrm{~min}$, 升温至 $85{ }^{\circ} \mathrm{C}$, 继续反应 $12 \mathrm{~h}$. 冷至室温后, 加入 $50 \mathrm{~mL}$ 


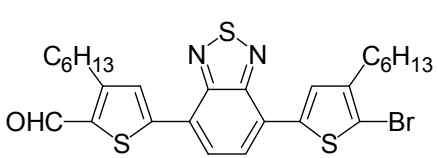

1

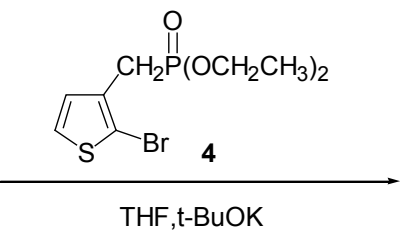

THF,t-BuOK

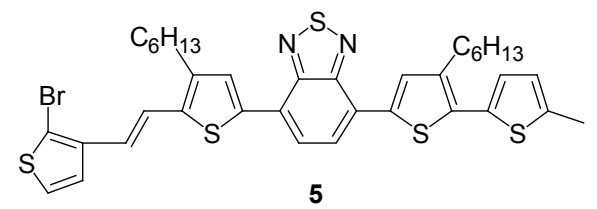

5

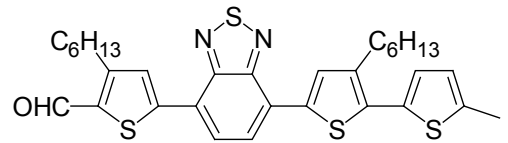

3

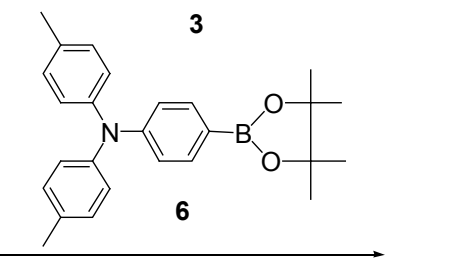

$\mathrm{Pd}\left(\mathrm{PPh}_{3}\right)_{4}$, Toluene, $\mathrm{K}_{2} \mathrm{CO}_{3}, 100^{\circ} \mathrm{C}, 72 \mathrm{~h}$

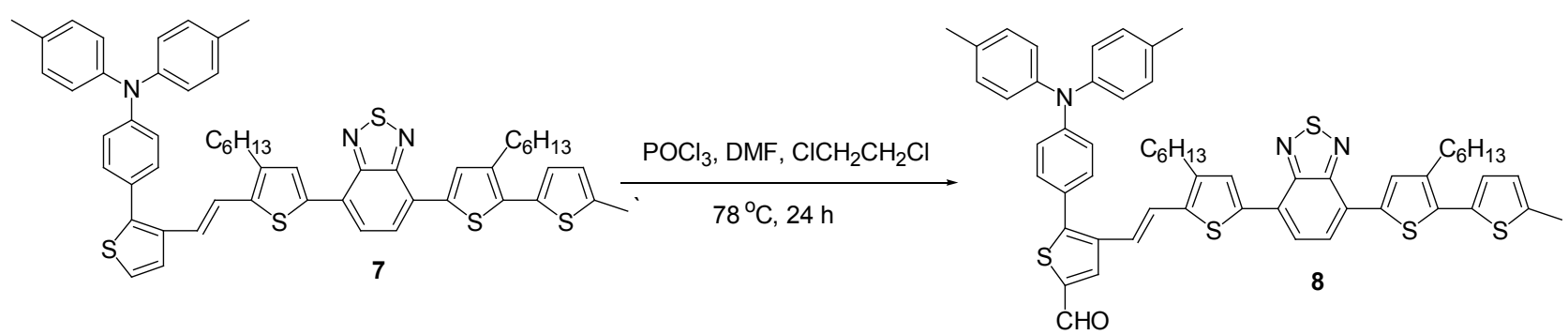

Piperidine, reflux, $24 \mathrm{~h}$

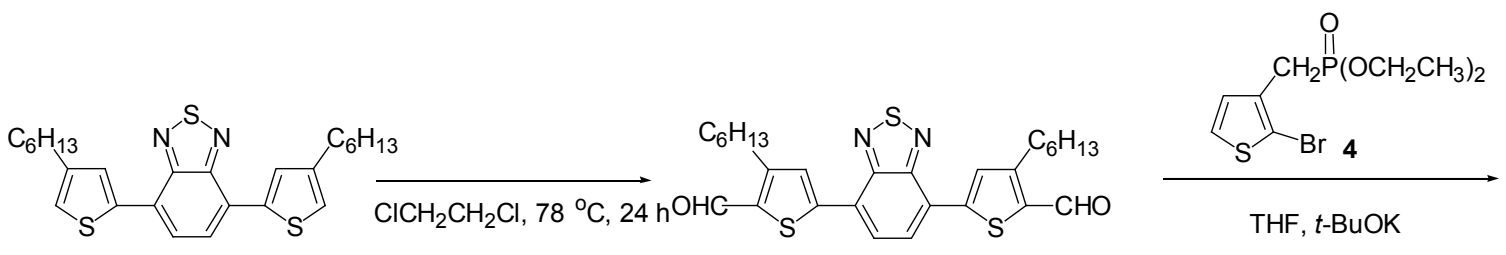

10<smiles>CCCCCCc1cc(-c2ccc(-c3cc(CCCCC)c(/C=C/c4ccsc4Br)s3)c3nsnc23)sc1/C=C/c1ccsc1Br</smiles>
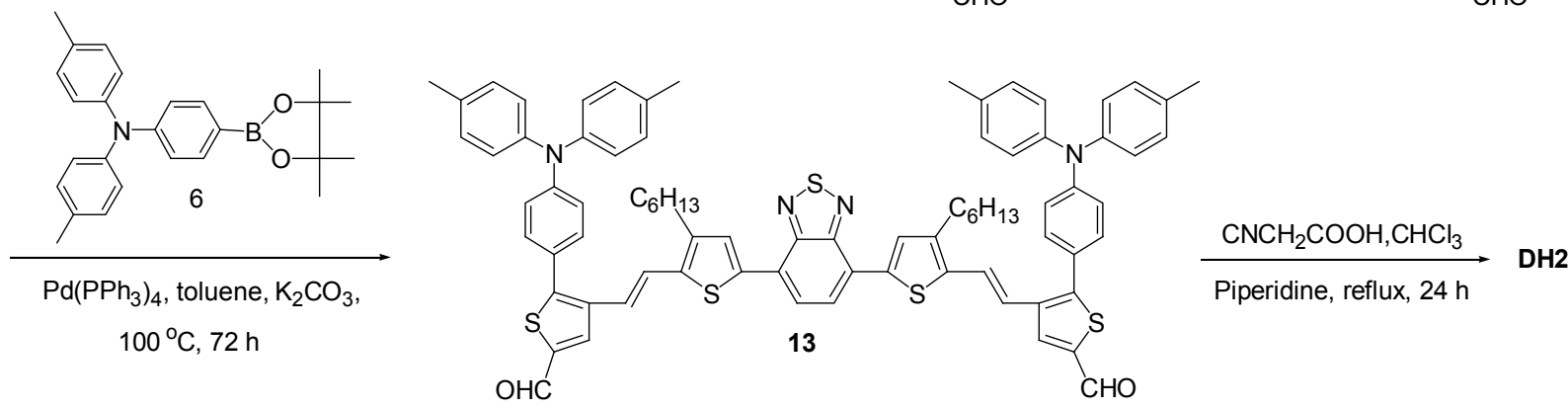

Scheme 2

饱和醋酸钠溶液水解 $1 \mathrm{~h}$, 混合液用二氯甲烷萃取, 并 用水洗涤三次, 有机相再用无水硫酸镁干燥 $12 \mathrm{~h}$. 过滤 后旋转蒸发溶剂, 用 $[V$ (石油醚) $: V($ 二氯甲烷 $)=1: 1]$ 作淋洗剂过硅胶柱, 得到紫色固体(0.2 g, 产率 48.5\%). $\left.{ }^{1} \mathrm{H} \mathrm{NMR} \mathrm{(CDCl} 3,400 \mathrm{MHz}\right) \delta: 9.68$ (s, 1H, CHO), 8.05 (s, $1 \mathrm{H}$, thienyl-H), 7.97 (s, $2 \mathrm{H}$, thienyl-H), $7.75(\mathrm{~d}, J=8 \mathrm{~Hz}$, $2 \mathrm{H}$, phenyl-H), $7.64\left(\mathrm{~d}, J=9 \mathrm{~Hz}, 1 \mathrm{H}, \mathrm{CH}_{2}=\mathrm{CH}_{2}\right), 7.42(\mathrm{~d}$, $\left.J=8 \mathrm{~Hz}, 1 \mathrm{H}, \mathrm{CH}_{2}=\mathrm{CH}_{2}\right), 7.21(\mathrm{~d}, J=10 \mathrm{~Hz}, 2 \mathrm{H}$, 
thienyl-H), $7.02 \sim 6.75(\mathrm{~m}, 12 \mathrm{H}$, phenyl-H), $2.82(\mathrm{t}, J=14$ $\left.\mathrm{Hz}, 2 \mathrm{H}, \mathrm{CH}_{2}\right), 2.53$ (t, $\left.J=4 \mathrm{~Hz}, 2 \mathrm{H}, \mathrm{CH}_{3}\right), 2.27(\mathrm{~s}, 6 \mathrm{H}$, $\left.\mathrm{CH}_{3}\right), 1.71 \sim 1.78\left(\mathrm{~m}, 4 \mathrm{H}, \mathrm{CH}_{2}\right), 1.24 \sim 1.42(\mathrm{~m}, 12 \mathrm{H}$, $\left.\mathrm{CH}_{2}\right), 0.90\left(\mathrm{t}, J=12 \mathrm{~Hz}, 6 \mathrm{H}, \mathrm{CH}_{3}\right)$. Anal calcd for $\mathrm{C}_{58} \mathrm{H}_{57} \mathrm{~N}_{3} \mathrm{OS}_{5}$ : C 71.64, H 5.91, N 4.32, S 16.49; found $\mathrm{C}$ 71.61, H 5.97, N 4.26, S 16.57.

\subsubsection{DD2 的合成}

在氩气保护下, 向 $100 \mathrm{~mL}$ 的两口瓶中依次加入 $0.20 \mathrm{~g}(0.21 \mathrm{mmol})$ 化合物 $\mathbf{8}, 0.17 \mathrm{~g}(2 \mathrm{mmol})$ 氧基乙酸, $10 \mathrm{~mL}$ 乙腈, 然后在 $\mathrm{Ar}$ 保护下用注射器注入 $0.2 \mathrm{~mL}$ 哌 啶, 回流反应 $24 \mathrm{~h}$. 冷却至室温, 用三氯甲烷萃取, 有 机相用 $0.1 \mathrm{~mol} / \mathrm{L}$ 的盐酸洗涤数次, 然后水洗 3 次, 用无 水硫酸镁干燥, 过滤, 旋干. 粗产品用 $[V$ (二氯甲烷) : $V($ 甲醇 $)=10: 1]$ 作洗脱剂过硅胶柱, 最后得到红色的 固体 $0.18 \mathrm{~g}$, 产率 $84.5 \%$. m.p. $103 \sim 105{ }^{\circ} \mathrm{C} ;{ }^{1} \mathrm{H}$ NMR $\left(\mathrm{CDCl}_{3}, 400 \mathrm{MHz}\right) \delta: 8.03(\mathrm{~s}, 1 \mathrm{H}$, thienyl-H), 7.97 (s, 2H, thienyl-H), 7.75 (d, $J=8 \mathrm{~Hz}, 2 \mathrm{H}$, phenyl-H), 7.64 (d, $J=9$ $\left.\mathrm{Hz}, 1 \mathrm{H}, \mathrm{CH}_{2}=\mathrm{CH}_{2}\right), 7.42\left(\mathrm{~d}, J=8 \mathrm{~Hz}, 1 \mathrm{H}, \mathrm{CH}_{2}=\mathrm{CH}_{2}\right)$, $7.21(\mathrm{~d}, J=10 \mathrm{~Hz}, 2 \mathrm{H}$, thienyl-H), $6.56 \sim 6.99(\mathrm{~m}, 12 \mathrm{H}$, phenyl-H), 2.96 (t, $J=14 \mathrm{~Hz}, 2 \mathrm{H}, \mathrm{CH}_{2}$ ), 2.66 (t, $J=8 \mathrm{~Hz}$, $\left.2 \mathrm{H}, \mathrm{CH}_{3}\right), 2.42\left(\mathrm{~s}, 6 \mathrm{H}, \mathrm{CH}_{3}\right), 1.82 \sim 2.18\left(\mathrm{~m}, 4 \mathrm{H}, \mathrm{CH}_{2}\right)$, $1.24 \sim 1.42\left(\mathrm{~m}, 12 \mathrm{H}, \mathrm{CH}_{2}\right), 0.87\left(\mathrm{t}, J=12 \mathrm{~Hz}, 6 \mathrm{H}, \mathrm{CH}_{3}\right)$; ${ }^{13} \mathrm{C} \mathrm{NMR}\left(\mathrm{CDCl}_{3}, 100 \mathrm{MHz}\right) \delta: 152.07,144.59,139.60$, $134.13,132.32,130.94,130.00,129.76,128.88,128.12$, $127.90,126.84,125.82,125.61,125.25,124.95,124.56$, $120.98,62.78,61.64,42.00,36.73,31.95,31.76,30.48$, $29.73,29.69,29.49,28.52,27.22,27.03,24.91,22.74$, 20.88, 15.31, 14.23, 14.14; MALDI-TOF MS calcd for $\mathrm{C}_{61} \mathrm{H}_{58} \mathrm{~N}_{4} \mathrm{O}_{2} \mathrm{~S}_{5}(\mathrm{M}+\mathrm{H})^{+}:$1039.324, found 1039.243. Anal calcd for $\mathrm{C}_{61} \mathrm{H}_{58} \mathrm{~N}_{4} \mathrm{O}_{2} \mathrm{~S}_{5}$ : C 70.48, H 5.62, N 5.39, S 15.42; found C 70.38, H 5.67, N 5.36, S 15.50.

3.2 .6 5-(7-(5-甲酰-4-已基噻吩-2-基)苯并 $[c][1,2,5]$ 塞 二唑-4-基)-3-已基噻吩-2-甲醛(10)的合成

合成方法与化合物 8 类似，投料改为 $4.69 \mathrm{~g}$ 化合物

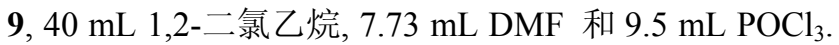
以 $[V($ 石油醚 $): V($ 二氯甲烷 $)=1: 1]$ 的溶剂作为洗脱剂 进行硅胶柱层析, 真空干燥后得红色固体 $4.38 \mathrm{~g}$, 产率 $83 \%$. ${ }^{1} \mathrm{H}$ NMR $\left(\mathrm{CDCl}_{3}, 400 \mathrm{MHz}\right) \delta: 10.13$ (s, 2H, CHO), 8.11 (s, 2H, phenyl-H), $8.03(\mathrm{~s}, 2 \mathrm{H}$, thienyl-H), $3.06(\mathrm{t}, J=$ $\left.14 \mathrm{~Hz}, 4 \mathrm{H}, \mathrm{CH}_{2}\right), 1.77 \sim 1.79\left(\mathrm{~m}, 4 \mathrm{H}, \mathrm{CH}_{2}\right), 1.42 \sim 1.49$ $\left(\mathrm{m}, 4 \mathrm{H}, \mathrm{CH}_{2}\right), 1.31 \sim 1.40\left(\mathrm{~m}, 8 \mathrm{H}, \mathrm{CH}_{2}\right), 0.92(\mathrm{t}, J=13 \mathrm{~Hz}$, $\left.6 \mathrm{H}, \mathrm{CH}_{3}\right) ;{ }^{13} \mathrm{C} \mathrm{NMR}\left(100 \mathrm{MHz}, \mathrm{CDCl}_{3}\right) \delta: 182.14,153.31$, $152.30,146.64,138.14,131.18,126.77,126.29,31.61$, 31.48, 29.08, 28.69, 22.60, 14.09; MALDI-TOF MS calcd for $\mathrm{C}_{28} \mathrm{H}_{32} \mathrm{~N}_{2} \mathrm{O}_{2} \mathrm{~S}_{3}(\mathrm{M}+\mathrm{H})^{+}:$525.170, found 525.190.
3.2.7 4,7-二(5-((E)-2-(2-溴噻吩-3-基)乙烯基)-4-已基 噻吩-2-基)苯并 $[c][1,2,5]$ 噻二唑(11)的合成

合成方法与化合物 5 类似，投料改为 $1.6 \mathrm{~g}$ 化合物 $4,1 \mathrm{~g}$ 化合物 $10,0.64 \mathrm{~g}$ 的 $t-\mathrm{BuOK}$, 以 [ $V$ (石油醚) : $V$ (二 氯甲烷 $)=3: 1]$ 的溶剂作为洗脱剂用硅胶柱进行层析, 最后得到紫红色固体 $1.43 \mathrm{~g}$, 产率 $89 \%$. ${ }^{1} \mathrm{H}$ NMR $\left(\mathrm{CDCl}_{3}, 400 \mathrm{MHz}\right) \delta: 7.98(\mathrm{~s}, 2 \mathrm{H}), 7.87(\mathrm{~s}, 2 \mathrm{H}), 7.26 \sim$ $7.29(\mathrm{~m}, 2 \mathrm{H}), 7.16 \sim 7.23(\mathrm{~m}, 4 \mathrm{H}), 7.01(\mathrm{~d}, J=16 \mathrm{~Hz}, 2 \mathrm{H})$, $2.73 \sim 2.77(\mathrm{~m}, 4 \mathrm{H}), 1.68 \sim 1.72(\mathrm{~m}, 4 \mathrm{H}), 1.25 \sim 1.43(\mathrm{~m}$, $12 \mathrm{H}), 0.90(\mathrm{t}, J=12 \mathrm{~Hz}, 6 \mathrm{H}) ;{ }^{13} \mathrm{C} \mathrm{NMR}\left(\mathrm{CDCl}_{3}, 100\right.$ MHz) $\delta: 152.55,142.92,138.29,137.50,137.04,130.62$, $126.06,125.46,125.31,124.62,121.88,120.57,111.05$, 31.73, 30.92, 29.20, 28.70, 22.66, 14.10; MALDI-TOF MS calcd for $\mathrm{C}_{38} \mathrm{H}_{38} \mathrm{Br}_{2} \mathrm{~N}_{2} \mathrm{~S}_{5} 841.998$, found 841.910.

$3.2 .84,4^{\prime}-\left(1 E, 1^{\prime} E\right)-\left(5,5^{\prime}-(\right.$ 苯并 $[c][1,2,5]$ 噻二唑-4,7-二 基)二(3-已基噻吩-5,2-二基)二(乙烯-1,2-二基)二(5-溴 噻吩-2-甲醛(12)的合成

合成方法与化合物 $\mathbf{8}$ 类似，将投料改为化合物 $\mathbf{1 1}$ $(0.50 \mathrm{~g}, 0.60 \mathrm{mmol}), 40 \mathrm{~mL}$ 的 1,2 -二氯乙烷, $0.56 \mathrm{~mL}$ 的 $\mathrm{DMF}, 0.48 \mathrm{~mL}$ 的 $\mathrm{POCl}_{3}$. 以二 $[V$ (石油醚) $: V($ 二氯甲 烷 $)=1: 1]$ 的溶剂为洗脱剂用硅胶柱进行层析, 最后得 到紫红色固体 $0.39 \mathrm{~g}$, 产率 $72.7 \% .{ }^{1} \mathrm{H} \mathrm{NMR}\left(\mathrm{CDCl}_{3}, 400\right.$ $\mathrm{MHz}) \delta: 9.74(\mathrm{~s}, 2 \mathrm{H}), 8.12(\mathrm{~s}, 2 \mathrm{H}), 7.83$ (s, 2H), $7.64(\mathrm{~s}$, 2H), 7.48 (d, $J=5 \mathrm{~Hz}, 2 \mathrm{H}), 6.82$ (d, $J=4 \mathrm{~Hz}, 2 \mathrm{H}), 2.88$ (t, $J=15 \mathrm{~Hz}, 4 \mathrm{H}), 1.73 \sim 1.77(\mathrm{~m}, 4 \mathrm{H}), 1.42 \sim 1.51(\mathrm{~m}, 4 \mathrm{H})$, $1.29 \sim 1.41(\mathrm{~m}, 8 \mathrm{H}), 0.92(\mathrm{t}, J=13 \mathrm{~Hz}, 6 \mathrm{H})$; MALDI-TOF MS calcd for $\mathrm{C}_{40} \mathrm{H}_{38} \mathrm{Br}_{2} \mathrm{~N}_{2} \mathrm{O}_{2} \mathrm{~S}_{5}$ 897.987, found 897.972.

$3.2 .94,4^{\prime}-\left(1 E, 1^{\prime} E\right)-\left(5,5^{\prime}-(\right.$ 苯并 $[c][1,2,5]$ 塞二唑-4,7-二 基)二(3-已基噻吩-5,2-二基)二(1,2-亚乙烯基)二 (5-(4-(二对苯甲基胺)苯基)噻吩-2-甲醛)(13)的合成

合成方法与化合物 7 类似，投料改为化合物 $6(0.37$ $\mathrm{g}, 0.33 \mathrm{mmol})$, 化合物 $12(0.37 \mathrm{~g}, 1 \mathrm{mmol}), 0.15 \mathrm{~g}$ 碳酸 钾, $40 \mathrm{~mL}$ 新蒸甲苯. 以 $V$ (石油醚 $): V($ 二氯甲烷 $)=$ $1: 1]$ 的溶剂作为洗脱剂用硅胶柱进行层析, 得到暗红 色固体 $0.31 \mathrm{~g}$, 产率 $73.8 \% .{ }^{1} \mathrm{H} \mathrm{NMR}\left(\mathrm{CDCl}_{3}, 400 \mathrm{MHz}\right)$ $\delta$ : 9.69 (s, 2H), 8.07 (s, 2H), 7.75 (s, 2H), 7.54 (s, 2H), 7.37 (d, $J=6 \mathrm{~Hz}, 2 \mathrm{H}), 7.33$ (d, $J=9 \mathrm{~Hz}, 8 \mathrm{H}), 7.21$ (d, $J=8$ $\mathrm{Hz}, 2 \mathrm{H}), 7.01$ (d, $J=8 \mathrm{~Hz}, 4 \mathrm{H}), 6.93$ (d, $J=8 \mathrm{~Hz}, 4 \mathrm{H})$, $6.84(\mathrm{~d}, J=9 \mathrm{~Hz}, 8 \mathrm{H}), 2.78(\mathrm{t}, J=15 \mathrm{~Hz}, 4 \mathrm{H}), 2.27$ (s, $12 \mathrm{H}), 1.32 \sim 1.39(\mathrm{~m}, 4 \mathrm{H}), 1.25 \sim 1.28(\mathrm{~m}, 12 \mathrm{H}), 0.90(\mathrm{t}$, $J=12 \mathrm{~Hz}, 6 \mathrm{H}) ;{ }^{13} \mathrm{C} \mathrm{NMR}\left(\mathrm{CDCl}_{3}, 100 \mathrm{MHz}\right) \delta: 192.39$, $152.38,150.63,147.86,144.84,144.60,143.32,135.73$, $134.28,133.24,132.87,130.28,129.87,129.74,128.67$, $127.95,126.60,126.55,126.20,125.82,125.24,125.00$, $124.83,121.34,120.50,31.61,31.19,31.15,29.70,29.18$, 
29.13, 28.67, 24.84, 22.62, 22.58, 20.79, 20.75, 14.09, 14.03. FT-IR (KBr) v: $1637(\mathrm{C}=\mathrm{O}), 997$ (trans-vinylene) $\mathrm{cm}^{-1}$; MALDI-TOF MS calcd for $\mathrm{C}_{80} \mathrm{H}_{74} \mathrm{~N}_{4} \mathrm{O}_{2} \mathrm{~S}_{5}$, 1282.440; found 1282.610. Anal calcd for $\mathrm{C}_{80} \mathrm{H}_{74} \mathrm{~N}_{4} \mathrm{O}_{2} \mathrm{~S}_{5}$ : C 74.85, H 5.81, N 4.36, S 12.49; found C 74.76, H 5.87, $\mathrm{N} 4.34, \mathrm{~S} 12.56$.

\subsubsection{DH2 的合成}

合成方法与 DD2 类似, 将投料改为化合物 $\mathbf{1 3}(0.25$ $\mathrm{g}, 0.20 \mathrm{mmol}), 0.2 \mathrm{~g}$ 氧基乙酸, $30 \mathrm{~mL}$ 新蒸乙腈和 $10 \mathrm{~mL}$ 三氯甲烷, $0.5 \mathrm{~mL}$ 哌啶, $50 \mathrm{~mL} 0.1 \mathrm{~mol} / \mathrm{L} \mathrm{HCl}$ 溶液. 以 $[V($ 二氯甲烷 $): V($ 石油醚 $)=10: 1]$ 为洗脱剂用硅胶柱层 析, 最后得到紫黑色固体 $0.17 \mathrm{~g}$, 产率 61.6\%. m.p. $104 \sim 106{ }^{\circ} \mathrm{C} ;{ }^{1} \mathrm{H}$ NMR $\left(\left(\mathrm{CD}_{3}\right)_{2} \mathrm{SO}, 400 \mathrm{MHz}\right) \delta: 8.26$ (s, 2H), 7.95 (s, 2H), $7.81(\mathrm{~s}, 2 \mathrm{H}), 7.66(\mathrm{~d}, J=6 \mathrm{~Hz}, 2 \mathrm{H}), 7.54$ (d, $J=9 \mathrm{~Hz}, 8 \mathrm{H}), 7.25$ (d, $J=8 \mathrm{~Hz}, 2 \mathrm{H}), 7.97$ (d, $J=8 \mathrm{~Hz}$, $8 \mathrm{H}), 6.80(\mathrm{~d}, J=8 \mathrm{~Hz}, 4 \mathrm{H}), 6.73(\mathrm{~d}, J=9 \mathrm{~Hz}, 8 \mathrm{H}), 2.71(\mathrm{t}$, $J=15 \mathrm{~Hz}, 4 \mathrm{H}), 2.15(\mathrm{~s}, 12 \mathrm{H}), 1.40 \sim 1.48(\mathrm{~m}, 4 \mathrm{H}), 1.27 \sim$ $1.33(\mathrm{~m}, 12 \mathrm{H}), 0.92$ (t, $J=12 \mathrm{~Hz}, 6 \mathrm{H})$; MALDI-TOF MS calcd for $\mathrm{C}_{86} \mathrm{H}_{76} \mathrm{~N}_{6} \mathrm{O}_{4} \mathrm{~S}_{5}(\mathrm{M}+\mathrm{H})^{+}:$1417.460, found 1417.641. Anal calcd for $\mathrm{C}_{86} \mathrm{H}_{76} \mathrm{~N}_{6} \mathrm{O}_{4} \mathrm{~S}_{5}$ : C 72.85, H 5.40, N 5.93, S 11.31; found C 72.78, H 5.46, N 5.88, S 11.38.

\section{3 染料吸附量的测量}

将吸附了有机染料的纳米晶 $\mathrm{TiO}_{2}$ 薄膜电极浸泡在 5 $\mathrm{mL}, 0.5 \mathrm{~mol} \cdot \mathrm{L}^{-1}$ 的 $\mathrm{NaOH}$ 乙醇/蒸馏水溶液中(乙醇和蒸 馏水的体积比为 $1: 1)$, 由于在碱性条件下, 染料分子 与 $\mathrm{TiO}_{2}$ 表面形成的酯键会发生水解, 吸附在电极表面 的有机染料会从 $\mathrm{TiO}_{2}$ 上脱附下来, 溶解于脱附液中. 浸 泡 $12 \mathrm{~h}$ 后, 采用紫外-可见分光光度计在室温下测量染 料脱附液的吸收光谱, 通过测量脱附液在某一波长的吸 光度来计算纳米晶 $\mathrm{TiO}_{2}$ 薄膜电极的染料吸附量 $c$ $\left(\mathrm{mol} \cdot \mathrm{cm}^{-2}\right)$. 染料的吸附量依式(1)计算:

$$
c=\frac{A V}{\varepsilon S}
$$

其中 $c$ 为染料的吸附量 $\left(\mathrm{mol} \cdot \mathrm{m}^{-2}\right), S$ 为电极表面涂覆有 $\mathrm{TiO}_{2}$ 薄膜区域的面积 $\left(\mathrm{cm}^{2}\right), A$ 为脱附液的吸光度, $V$ 为脱 附液的体积 $(\mathrm{L}), \varepsilon$ 为染料在脱附液中的摩尔消光系数.

\section{4 染料敏化太阳能电池器件的制备和光伏性能测试}

$\mathrm{TiO}_{2}$ 胶体 $\mathrm{A}, \mathrm{B}$ 的制备参考文献[37], 将含氟的氧化 锡导电玻璃(FTO)清洗干净后, 置于 $0.04 \mathrm{~mol} / \mathrm{L}$ 的 $\mathrm{TiCl}_{4}$ 水溶液中 $70{ }^{\circ} \mathrm{C}$ 保温 $30 \mathrm{~min}$, 取出用蒸馏水和无水乙醇 依次冲洗后, 置于马弗炉中 $450{ }^{\circ} \mathrm{C}$ 下煅烧 $30 \mathrm{~min}$. 在处 理好的导电玻璃上用玻璃棒刮涂一层 $10 \sim 15 \mu \mathrm{m} \mathrm{TiO}_{2}$ 胶体 $\mathrm{A}$, 待干燥后继续刮涂一层 $4 \sim 6 \mu \mathrm{m}$ 的 $\mathrm{TiO}_{2}$ 胶体 $\mathrm{B}$, 然后置于马弗炉中在 $450{ }^{\circ} \mathrm{C}$ 下煅烧 $30 \mathrm{~min}$, 用 40 $\mathrm{mmol} / \mathrm{L}$ 的 $\mathrm{TiCl}_{4}$ 水溶液 $70{ }^{\circ} \mathrm{C}$ 处理 $30 \mathrm{~min}$, 再次在 450 ${ }^{\circ} \mathrm{C}$ 下煅烧 $30 \mathrm{~min}$, 待冷却到 $80{ }^{\circ} \mathrm{C}$ 时将其浸入到含有 0.5 $\mathrm{mmol} / \mathrm{L}$ 染料的溶液中, 室温避光浸泡 $12 \mathrm{~h}$ 后取出, 用 无水乙醇冲洗后烘干, 在浸泡有染料的电极表面滴加液 态电解质, 以铂片作为对电极组装成染料敏化太阳电池 器件, 电解质组成为 $0.5 \mathrm{~mol} / \mathrm{L} \mathrm{LiI}, 0.05 \mathrm{~mol} / \mathrm{L} \mathrm{I}_{2}, 0.5$ $\mathrm{mol} / \mathrm{L} \mathrm{TBP}, 0.6 \mathrm{~mol} / \mathrm{L} 1$-甲基-3-己基咪唑碘(MHII)的 3甲氧基丙腈溶液.

光伏测试采用 $500 \mathrm{~W}$ 氙灯与 $\mathrm{AM} 1.5 \mathrm{G}$ 滤光片组合 作为模拟太阳光的白光光源, 在 $100 \mathrm{~mW} / \mathrm{cm}^{2}$ 光强下测 试其光伏性能, 电池有效光照面积为 $0.196 \mathrm{~cm}^{2}, J-V$ 曲 线和 IPCE 使用 Keithley 2602 和 Zolix Omni- $\lambda 150$ 单色 仪进行测量, 并通过计算机进行控制.

\section{References}

[1] O'Regan, B.-C.; Grätzel, M. Nature 1991, 335, 7377.

[2] Wang, J.-W.; Li, Y.; Xu, Y.-L.; Li, Y.; Shen, K.-H. Acta Chim. Sinica 2012, 70, 1278 (in Chinese).

(王纪伟, 李莹, 徐艳玲, 李杨, 申凯华, 化学学报, 2012, 70, 1278.)

[3] Mao, L.; Xu, Y.-J.; Wang, X.-D.; Liu, X. -J.; Sun, Z.; Xue, S. Acta Chim. Sinica 2011, 69, 2092 (in Chinese).

(梁茂, 徐英军, 王旭达, 刘秀杰, 孙喆, 薛松, 化学学报, 2011, 69, 2092.)

[4] Feng, X.-M.; Huang, X. -W.; Tan, Z.; Zhao, B.; Tan, S.-T. Acta Chim. Sinica 2011, 69, 653 (in Chinese).

(冯小明, 黄先威, 谭卓, 赵斌, 谭松庭, 化学学报, 2011, 69, 653.)

[5] He, J.-J.; Chen, S.-X.; Wang, T.-T.; Zeng, H.-P. Chin. J. Org. Chem. 2012, 472 (in Chinese).

(何俊杰, 陈舒欣, 王婷婷, 曾和平, 有机化学, 2012, 32, 472.)

[6] Wang, Z.-Y.; Wang, R.-Y.; Fu, N.-Y. Chin. J. Org. Chem. 2011, 31, 415 (in Chinese). (王振宇, 王如勇, 傅南雁, 有机化学, 2011, 31, 415.)

[7] Yella, A.; Lee, H.; Tsao, H.-N.; Yi, C.; Chandiran, A.-K.; Nazeeruddin, M.-K.; Diau, E.-W.; Yeh, C.; Zakeeruddin, S.-M.; Grätzel, M. Science 2011, 334, 629.

[8] Nazeeruddin, M.-K.; De Angelis, F.; Fantacci, S.; Selloni, A.; Viscardi, G.; Liska, P.; Ito, S.; Takeru, B.; Grätzel, M. J. Am. Chem. Soc. 2005, 127, 16835.

[9] Gao, F.; Wang, Y.; Shi, D.; Zhang, J.; Wang, M.; Jing, X.; Humphry-Baker, R.; Wang, P.; Zakeeruddin, S.-M.; Grätzel, M. J. Am. Chem. Soc. 2008, 130, 10720.

[10] Wang, Q.; Ito, S.; Grätzel, M.; Fabregat-Santiago, F.; Mora-Sero, I.; Bisquert, J.; Bessho, T.; Imai, H. J. Phys. Chem. B 2006, 110, 25210.

[11] Chiba, Y.; Islam, A.; Watanabe, Y.; Komiya, R.; Koide, N.; Han, L. Jpn. J. Appl. Phys. 2006, 45, L638.

[12] Zeng, W.; Cao, Y.; Bai, Y.; Wang, Y.; Shi, Y.; Zhang, M.; Wang, F.; Pan, C.; Wang, P. Chem. Mater. 2010, 22, 1915.

[13] Cai, S.; Hu, X.; Zhang, Z.; Su, J.; Li, X.; Islam, A.; Han, L.; Tian, H. J. Mater. Chem. A 2013, 1, 4763.

[14] Feng, Q.; Jia, X.; Zhou, G.; Wang, Z. Chem. Commun. 2013, 49, 7445.

[15] Liu, B.; Liu, Q.; You, D.; Li, X.; Naruta, Y.; Zhu, W. J. Mater. Chem. 2012, 22, 13348. 
[16] Li, L.; Hao, Y.; Yang, X.; Zhao, J.; Tian, H.; Teng, C.; Anders, H.; Licheng, S. ChemSusChem. 2011, 4, 609.

[17] Tang, X.; Wang, Y.-X Acta Chim. Sinica 2013, 71, 193 (in Chinese). (唐笑, 汪禹汛, 化学学报, 2013, 71, 193.)

[18] Huang, X.-W.; Deng, J.-Y.; Xu, L.; Shen, P.; Zhao, B.; Tan, S.-T. Acta Chim. Sinica 2012, 70, 1604 (in Chinese).

(黄先威, 邓继勇, 许律, 沈平, 赵斌, 谭松庭, 化学学报, 2012, 70, 1604.)

[19] Abbotto, A.; Leandri, V.; Manfredi, N.; De Angelis, F.; Pastore, M.; Yum, J.; Nazeeruddin, M.-K.; Grätzel, M. Eur. J. Org. Chem. 2011, 2011, 6195.

[20] Sirohi, R.; Kim, D.-H.; Yu, S.; Lee, S.-H. Dyes Pigm. 2012, 92, 1132.

[21] Park, S.-S.; Won, Y.-S.; Choi, Y.-C.; Kim, J.-H. Energy Fuels 2009, 23, 3732.

[22] Yen, Y.; Chen, W.; Hsu, C.; Chou, H.; Lin, J.-T.; Yeh, M.-P. Org. Lett. 2011, 13, 4930.

[23] Ji, Z.; Natu, G.; Huang, Z.; Wu, Y Energy Environ. Sci. 2011, 4, 2818.

[24] Heredia, D.; Natera, J.; Gervaldo, M.; Otero, L.; Fungo, F.; Lin, C.; Wong, K. Org. Lett. 2009, 12, 12.

[25] Li, Q.; Shi, J.; Li, H.; Li, S.; Zhong, C.; Guo, F.; Peng, M.; Hua, J.; Qin, J.; Li, Z. J. Mater. Chem. 2012, 22, 6689.

[26] Jiang, S.-H.; Zhou, W.-P.; Tan, Z.; Shen, P.; Zhao, B.; Tan, S.-T. Sci. Sin. Chim. 2011, 41, 982 (in Chinese).
(蒋生辉, 周维平, 谭卓, 沈平, 赵斌, 谭松庭, 中国科学, 2011, 41, 982.)

[27] Wu, W.; Hua, J.; Jin, Y.; Zhan, W.; Tian, H. Photochem. Photobiol. Sci. 2008, 7, 63.

[28] Roquet, S.; Cravino, A.; Leriche, P.; Alévêque, O.; Frère, P.; Roncali, J. J. Am. Chem. Soc. 2006, 128, 3459.

[29] Chen, R.; Yang, X.; Tian, H.; Wang, X.; Hagfeldt, A.; Sun, L. Chem. Mater. 2007, 19, 4007.

[30] Tachibana, Y.; Hara, K.; Sayama, K.; Arakawa, H. Chem. Mater. 2002, 14, 2527.

[31] O'Regan, B.-C.; López-Duarte, I.; Martínez-Díaz, M.-V.; Forneli, A.; Albero, J.; Morandeira, A.; Palomares, E.; Torres, T.; Durrant, J.-R. J. Am. Chem. Soc. 2008, 130, 2906.

[32] O'Regan, B.-C.; Walley, K.; Juozapavicius, M.; Anderson, A.; Matar, F.; Ghaddar, T.; Zakeeruddin, S.-M.; Klein, C.; Durrant, J.-R. J. Am. Chem. Soc. 2009, 131, 3541.

[33] Jena, A.-K.; Bhargava, P. RSC Adv. 2013, 3, 2655.

[34] Gu, Z.; Deng, L.; Luo, H.; Guo, X.; Li, H.; Cao, Z.; Liu, X.; Li, X.; Huang, H.; Tan, Y.; Pei, Y.; Tan, S. J. Polym. Sci., Polym. Chem. 2012, 50, 3848.

[35] Sasaki, K.; Tokuda, O.; Hirota, T.; Luo, J.; Castle, R.-N. J. Heterocycl. Chem. 1996, 33, 847.

[36] Zhang, S.; Guo, Y.; Fan, H.; Liu, Y.; Chen, H.; Yang, G.; Zhan, X.; Liu, Y.; Li, Y.; Yang, Y. J. Polym. Sci., Polym. Chem. 2009, 47(20), 5498 .

(Li, L.; Lu, Z.) 